\title{
Exploiting Symmetry in the Power Flow Equations Using Monodromy
}

\author{
Julia Lindberg ${ }^{1}$, Nigel Boston ${ }^{1,2}$, Bernard C. Lesieutre ${ }^{1}$ \\ Department of Electrical and Computer Engineering ${ }^{1}$, Department of Mathematics ${ }^{2}$ \\ University of Wisconsin-Madison \\ Madison, USA, 53706 \\ jrlindberg@wisc.edu, nboston@wisc.edu, lesieutre@wisc.edu
}

\begin{abstract}
We propose solving the power flow equations using monodromy. We prove the variety under consideration decomposes into trivial and nontrivial subvarieties and that the nontrivial subvariety is irreducible. We also show various symmetries in the solutions. We finish by giving numerical results comparing monodromy against polyhedral and total degree homotopy methods and giving an example of a network where we can find all solutions to the power flow equation using monodromy where other homotopy techniques fail. This work gives hope that finding all solutions to the power flow equations for networks of realistic size is possible.
\end{abstract}

\section{Introduction}

The optimal power flow problem is a nonconvex quadratically constrained quadratic program that seeks to minimize generation costs of electricity subject to demand constraints, as well as physical and engineering constraints. The power flow equations are a system of quadratic equations that give the nonconvex constraints in the optimal power flow problem. Recent work has used techniques from algebraic geometry to find all solutions to the power flow equations for a fixed network [1, 2, 3], as well as study the distribution of the number of real solutions [4, 5, 6]. While there has been some success using numerical and symbolic techniques, these methods don't take advantage of any of the symmetry in the power flow equations. In this paper we apply monodromy techniques to find all complex solutions to the power flow equations for networks of varying size.

\section{The Power Flow Equations}

We model an $n$-node electric power network as a biconnected 1 , undirected graph, $G=(V, E)$, where each vertex represents a node in the power network. There is an edge, $e_{k m}$ between vertices $v_{k}$ and $v_{m}$ if the corresponding nodes in the power network are connected. Each edge has a known

\footnotetext{
${ }^{1} \mathrm{~A}$ graph is biconnected if removing any vertex does not disconnect the graph. For a graph that is not biconnected, the power flow equations decouple so they are analyzed separately on smaller graphs.
} 
susceptance $b_{k m} \in \mathbb{R}$. At each node, $k$, the relationship between the active power flows is captured by the nonlinear relations

$$
\begin{aligned}
x_{k}^{2}+y_{k}^{2} & =1 \\
\sum_{m=0}^{n-1} b_{k m}\left(x_{k} y_{m}-x_{m} y_{k}\right) & =P_{k}
\end{aligned}
$$

where $P_{k} \in \mathbb{R}$ are the active power injections for $k=1, \ldots, n-1$. We fix $v_{0}$ to be the reference node, meaning $x_{0}=1$ and $y_{0}=0$. Under these assumptions our power system is lossless and it has all $P V$ nodes. We assume our network has zero power injections so $P_{k}=0$ for all $k=1, \ldots, n-1$. Under these assumptions the parameters are the susceptances $b_{k m}$ and the variables are $x_{k}, y_{k}$ for $k=1, \ldots, n-1$. For fixed $b_{k m} \in \mathbb{R}$ we aim to find all complex solutions to (2.1).

\section{Symmetry in Solutions}

We briefly outline the idea of monodromy below using the same notation as [7], but give [7, 8, 9], as more complete references. Let $F_{b}$ be a parameterized polynomial system in $N$ variables and call the space of all such polynomial systems $B$. Assume the solution set of $F_{b}$ is zero dimensional. Let $\mathcal{V}$ denote the solution variety of $F_{b}$ i.e. $\mathcal{V}=\left\{\left(F_{b}, x\right) \in B \times \mathbb{C}^{N}: F_{b}(x)=0\right\}$. Consider the projection $\pi: \mathcal{V} \rightarrow B$ that maps a pair $\left(F_{b}, x\right) \mapsto F_{b}$ and the fibers $\pi^{-1}\left(F_{b}\right)=\left\{x \in \mathbb{C}^{N}: F_{b}(x)=0\right\}$. For almost all choices of parameters in $B,\left|\pi^{-1}\left(F_{b}\right)\right|=K$ is constant. Define $D$ to be the discriminant locus of $F_{b}$, this is the set of measure zero in $B$ where $\left|\pi^{-1}\left(F_{b}\right)\right| \neq K$. We define the fundamental group $\pi_{1}(B \backslash D)$ as a set of loops modulo homotopy equivalence that start and finish at a point $b \in B \backslash D$. Each loop permutes elements in $\pi^{-1}\left(F_{b}\right)$ and induces a group action called the monodromy action. Monodromy methods work by taking one solution $\hat{x}$ to the system of equations $F_{\hat{b}}$ and finding other elements of $\pi^{-1}\left(F_{\hat{b}}\right)$ via the monodromy action. The monodromy action is transitive if and only if the variety $V\left(F_{b}\right)$ is irreducible.

Under the assumptions given in Section 2, for any graph $G$, equations (2.1) have $2^{n-1}$ trivial solutions of the form $\left(x_{k}, y_{k}\right)=( \pm 1,0)$. Therefore, our problem is reduced to finding all nontrivial solutions of (2.1). In order to apply monodromy and have any hope of finding all nontrivial solutions we need the nontrivial solutions to form an irreducible variety.

Lemma 3.1. The nontrivial solutions of (2.1) form an irreducible variety.

Proof. By Theorem 6 of [10], the nontrivial component of (2.1) for tree networks is empty, so this

statement is vacuously true. Consider the change of variables $x_{i}=\frac{2 t_{i}}{1+t_{i}^{2}}$ and $y_{i}=\frac{1-t_{i}^{2}}{1+t_{i}^{2}}$. This gives a new system of equations for $k=1, \ldots, n-1$

$$
0=\sum_{m=0}^{n-1} b_{k m}\left(\frac{2 t_{k}\left(1-t_{m}^{2}\right)-2 t_{m}\left(1-t_{k}^{2}\right)}{\left(1+t_{k}^{2}\right)\left(1+t_{m}^{2}\right)}\right) .
$$

By Remark 2 of [7] is suffices to show that the following map has dense image:

$$
\begin{gathered}
\pi: \mathcal{V} \rightarrow \mathbb{C}^{n-1} \\
\left(F_{b}, t\right) \mapsto t
\end{gathered}
$$


where $F_{b}$ is the system of equations defined in (3.1) and $t=\left(t_{1}, \ldots, t_{n-1}\right)$. For all $t_{k} \in \mathbb{C} \backslash\{ \pm \sqrt{-1}, 0, \pm 1\}$, $k=1, \ldots, n-1$, this gives a linear system of $n-1$ equations in $|E|$ unknowns where the unknowns are the susceptances $b_{k m}$. Since we do not consider trees, $|E| \geq n$. Let $b \in \mathbb{R}^{|E|}$ be the vector of susceptances. Then this linear system can be written as $A b=0$ where $A \in \mathbb{C}^{n-1 \times|E|}$ is a weighted incidence matrix of $G$ with the first row removed. This matrix has rank $n-1$ so long as none of the weights are zero, which occurs for all $t_{k}, t_{m} \notin\{ \pm \sqrt{-1}, 0, \pm 1\}, t_{k} \neq t_{m}$. Therefore, for generic $t \in \mathbb{C}^{n-1}$ we can find a nonzero solution $b$ to (3.1) giving that the map $\left(F_{b}, t\right) \mapsto t$ is dense in $\mathbb{C}^{n-1}$.

In addition to ignoring the trivial component, we also wish to exploit the symmetry of (2.1).

Lemma 3.2. If $\left(x_{1}, \ldots, x_{n-1}, y_{1}, \ldots, y_{n-1}\right)$ is a solution to (2.1), so is $\left(x_{1}, \ldots, x_{n-1},-y_{1}, \ldots,-y_{n-1}\right)$ Proof. Substituting in $\left(x_{1}, \ldots, x_{n-1},-y_{1}, \ldots,-y_{n-1}\right)$ to (2.1) the result is immediate.

Lemma 3.3. Let $G=(V, E)$ be a bipartite graph with disjoint vertex sets $S, T \subset V$ that partition $V$ where for all $e=v_{m} v_{n} \in E, v_{m} \in S$ and $v_{n} \in T$. Without loss of generality, say $v_{1}, \ldots, v_{s} \in S$ and $v_{s+1}, \ldots, v_{n-1} \in T$. If $\left(x_{1}, \ldots, x_{n-1}, y_{1}, \ldots, y_{n-1}\right)$ is a solution to (2.1) so is

1. $\left(x_{1}, \ldots, x_{n-1},-y_{1}, \ldots,-y_{n-1}\right)$

2. $\left(-x_{1}, \ldots,-x_{s}, x_{s+1}, \ldots, x_{n-1}, y_{1}, \ldots, y_{s},-y_{s+1}, \ldots,-y_{n-1}\right)$

3. $\left(-x_{1}, \ldots,-x_{s}, x_{s+1}, \ldots, x_{n-1},-y_{1}, \ldots,-y_{s}, y_{s+1}, \ldots, y_{n-1}\right)$

Proof. At a node $k \in S$ the power flow equations are

$$
0=\sum_{m=s+1}^{n-1} b_{k m}\left(x_{m} y_{k}-x_{k} y_{m}\right) \text {. }
$$

At a node $l \in T$ the power flow equations are

$$
0=\sum_{m=1}^{s} b_{l m}\left(x_{m} y_{l}-x_{l} y_{m}\right) .
$$

Substituting in $(1)-(3)$ to the two expressions above, the result is clear.

\section{Numerical Simulations}

For all numerical computations we use the package HomotopyContinuation.j1 [11. Tables 1 and 2 show the average number of loops tracked to find all solutions using monodromy in comparison with the number of paths needed to track for polyhedral and total degree homotopy continuation methods. These tables also show the time it took to find all solutions. In each case, monodromy is the fastest method to find all solutions.

Remark 4.1. Another advantage to monodromy is that we don't lose solutions as we track them from a start to target system. We observed in small networks that all methods found all solutions but for larger networks polyhedral and total degree homotopy methods lost solutions. 


\begin{tabular}{|c|c|c|c|c|c|c|c|}
\hline & $K_{4}$ & $K_{5}$ & $K_{6}$ & $K_{7}$ & $K_{8}$ & $K_{9}$ & $K_{10}$ \\
\hline \# of loops: monodromy & 24.4 & 94.8 & 470.4 & 1669.2 & 7915 & 25112.2 & 95829.2 \\
\# of paths: polyhedral & 40 & 192 & 864 & 3712 & 15488 & 63488 & 257536 \\
\# of paths: total degree & 64 & 256 & 1024 & 4096 & 16384 & 65536 & 262144 \\
\hline time (s): monodromy & 0.01 & 0.05 & 0.37 & 1.97 & 16.39 & 65.33 & 357.926 \\
time (s): polyhedral & 0.06 & 0.37 & 2.53 & 17.10 & 112.43 & 609.49 & 2637.22 \\
time (s): total degree & 0.04 & 0.21 & 1.45 & 8.17 & 48.78 & 329.60 & 1510.01 \\
\hline
\end{tabular}

Table 1: Numerical results to find all solutions for complete networks

\begin{tabular}{|c|c|c|c|c|c|c|}
\hline & $C_{5}$ & $C_{6}$ & $C_{7}$ & $C_{8}$ & $C_{9}$ & $C_{10}$ \\
\hline \# of loops: monodromy & 181.6 & 176.6 & 962.0 & 921.6 & 1110.6 & 752 \\
\# of paths: polyhedral & 80 & 256 & 832 & 2688 & 8704 & 28160 \\
\# of paths: total degree & 256 & 1024 & 4096 & 16384 & 65536 & 262144 \\
\hline time (s): monodromy & 0.13 & 0.158 & 1.10 & 1.46 & 2.48 & 2.60 \\
time (s): polyhedral & 2.7 & 3.03 & 5.37 & 14.8 & 56.36 & 211.24 \\
time (s): total degree & 2.11 & 3.40 & 9.76 & 31.91 & 200.41 & 862.50 \\
\hline
\end{tabular}

Table 2: Numerical results to find all solutions for cyclic networks

Example 4.2. A final benefit of monodromy when applied to the power flow equations is that it can find all complex solutions when other methods can't. We consider the cyclic graph on 20 vertices. This system has 1,847,560 complex solutions but ignoring the trivial solutions and up to symmetry it has 330,818 . If we tried to use total degree homotopy on this system, the Bezout bound is $274,877,906,944$ so we would have to track over 274 billion paths. In addition, polyhedral methods aren't practical as the solver could not find a start system. Using monodromy we found all 330, 818 solutions in 15,375 seconds after tracking 792,934 loops. This example is the largest network to the authors' knowledge for which all solutions to the power flow equations have been found for a power system model 2

\section{Conclusion}

In this note we applied monodromy methods to the power flow equations with great results. Monodromy methods gain tremendous computational speed-up by decomposing the variety into trivial and nontrivial components and solving up to symmetry. Finally, we are able to push the current computational limits and find all solutions to the power flow equations for the cylic graph on 20 nodes, the largest power network in which all solutions have been found to date.

\section{Acknowledgements}

The authors' gratefully thank Jose Israel Rodriguez for his helpful comments and insight.

\footnotetext{
${ }^{2}$ The authors' note that in [12] all real solutions to a network on 60 vertices were found, but as noted by the authors in [12, the assumptions in that paper are not attainable by any realistic power systems model.
} 


\section{References}

[1] D. Mehta, H. D. Nguyen, and K. Turitsyn. Numerical polynomial homotopy continuation method to locate all the power flow solutions. IEEE Transactions on Power Systems, 10(12):2972-2980, 2016.

[2] Alisha Zachariah and Zachary Charles. Efficiently finding all power flow solutions to tree networks. In 201755 th Annual Allerton Conference on Communication, Control, and Computing (Allerton), pages 1107-1114, 2017.

[3] Tianran Chen and Dhagash Mehta. On the network topology dependent solution count of the algebraic load flow equations. IEEE Transactions on Power Systems, 33(2):1451-1460, 2018.

[4] Julia Lindberg, Alisha Zachariah, Nigel Boston, and Bernard Lesieutre. The geometry of real solutions to the power flow equations. In 2018 56th Annual Allerton Conference on Communication, Control, and Computing (Allerton), pages 596-603, 2018.

[5] Bernard Lesieutre, Julia Lindberg, Alisha Zachariah, and Nigel Boston. On the distribution of real-valued solutions to the power flow equations. In 2019 57th Annual Allerton Conference on Communication, Control, and Computing (Allerton), pages 165-170, 2019.

[6] Alisha Zachariah, Zachary Charles, Nigel Boston, and Bernard Lesieutre. Distributions of the number of solutions to the network power flow equations. In 2018 IEEE International Symposium on Circuits and Systems (ISCAS), pages 1-5, 2018.

[7] Timothy Duff, Cvetelina Hill, Anders Nedergaard Jensen, Kisun Lee, Anton Leykin, and Jeff Sommars. Solving polynomial systems via homotopy continuation and monodromy. IMA Journal of Numerical Analysis, 39(3):1421-1446, 2019.

[8] Carlos Améndola and Jose Israel Rodriguez. Solving parameterized polynomial systems with decomposable projections. arXiv preprint arXiv:1612.08807, 2016.

[9] Abraham Martín del Campo and Jose Israel Rodriguez. Critical points via monodromy and local methods. Journal of Symbolic Computation, 79:559-574, 2017.

[10] Tianran Chen, Robert Davis, and Dhagash Mehta. Counting equilibria of the Kuramoto model using birationally invariant intersection index. SIAM Journal on Applied Algebra and Geometry, 2(4):489-507, 2018.

[11] Paul Breiding and Sascha Timme. Homotopycontinuation.jl: A package for homotopy continuation in Julia. In International Congress on Mathematical Software, pages 458-465. Springer, 2018.

[12] Owen Coss, Jonathan D. Hauenstein, Hoon Hong, and Daniel K. Molzahn. Locating and counting equilibria of the Kuramoto model with rank-one coupling. SIAM Journal on Applied Algebra and Geometry, 2(1):45-71, 2018. 